\title{
Spin edge states: An exact solution and oscillations of the spin current
}

\author{
V. L. Grigoryan, ${ }^{1}$ A. Matos Abiague, ${ }^{2}$ and S. M. Badalyan ${ }^{1,2, *}$ \\ ${ }^{1}$ Department of Radiophysics, Yerevan State University, 1 A. Manoukian Street, 375025 Yerevan, Armenia \\ ${ }^{2}$ Department of Physics, University of Regensburg, 93040 Regensburg, Germany \\ (Received 14 August 2009; revised manuscript received 24 September 2009; published 15 October 2009)
}

\begin{abstract}
We study the spin edge states, induced by the combined effect of spin-orbit interaction (SOI) and hard-wall confining potential, in a two-dimensional electron system, exposed to a perpendicular magnetic field. We find an exact solution of the problem and show that the spin-resolved edge states are separated in space. The SOI-generated rearrangement of the spectrum results in a peaked behavior of the net-spin current versus the Fermi energy. The predicted oscillations of the spin current with a period, determined by the SOI-renormalized cyclotron energy, can serve as an effective tool for controlling the spin motion in spintronic devices.
\end{abstract}

DOI: 10.1103/PhysRevB.80.165320 PACS number(s): 73.21.Fg, 73.63.Hs, 72.25.Dc, 72.10. $-\mathrm{d}$

\section{INTRODUCTION}

The principal importance of spin-orbit interaction (SOI) is in its ability to link the electron charge and spin degrees of freedom, which is fertile for novel physical phenomena. ${ }^{1-3}$ Unlike the charge, the electron spin is double valued and identifies two system components, which can be separated as in the spin-Hall effect ${ }^{4,5}$ or mixed via the spin-Coulomb drag. ${ }^{6,7}$ There are different mechanisms, realizing SOI, ${ }^{1}$ and the interplay between them produces another rich arena for study and potential applications in spintronics. ${ }^{8,9}$

In two-dimensional electron systems (2DES) of the quantum-Hall-effect geometry, the extended edge state play a central role in understanding of transport phenomena. ${ }^{10-12}$ The suppression of backscattering and interedge-state relaxation ${ }^{13-15}$ make possible nondissipative transport through edge channels. In the presence of SOI the nonlocal transport through spin-polarized edge channels holds promise of providing even richer phenomenology and greater power of electronic applications. Another strong motivation for investigations of spin edge states is related to the recent experimental realization of the Mach-Zehnder. ${ }^{16}$

Recently several theoretical papers have addressed the effect of SOI on the edge states in restricted 2DES (Refs. 17-21) and along magnetic interfaces. ${ }^{22,23}$ All these works, however, find unlikely an exact analytical solution of the edge state problem and adopt different numerical approaches, ${ }^{18-20,22,23}$ use a parabolic confining potential, ${ }^{21}$ which has no flat domain, or give an analytical approximation in the limit of strong magnetic fields ${ }^{17}$ where the effective SOI coupling is small.

Here we present an analytical solution to the spin edge states, induced by the combined effect of SOI and hard-wall confining potential. We derive an exact formula for the electron energy dispersions and calculate their spectral and transport properties. We find that due to SOI the spin edge states are resolved not only in the energy but are also separated in space: an effect, which is not captured by the approximate approach, adopted in Ref. 17. From the energy spectrum we calculate the electron-group velocity and the average spin components. We find that the magnitude of spin components are not equal in the up and down states. In the quasibulk states the electron spins are mainly aligned along the mag- netic field while near the hard wall the spins of edge states become mainly perpendicular to their propagation direction. Using these ingredients we calculate the components of the net-spin current as a function of the Fermi energy and show that the SOI-induced splitting results in the peaked behavior of the spin current. We argue that the predicted oscillations of the spin current with a period, determined by the renormalized cyclotron frequency, can serve as a new tool for manipulating spin currents in a controllable manner. The developed approach here is equally applicable to the spin edge states along magnetic interfaces in nonhomogeneous magnetic fields.

\section{THEORETICAL CONCEPT}

We assume that the 2DES resides in a quantum well, formed in the (001) plane of a zincblende semiconductor heterostructure, and is exposed to a perpendicular homogeneous magnetic field, $\vec{B}=B_{0} \hat{\mathbf{z}}$. The motion of electrons in the 2DES is confined by an infinite hard-wall potential, $V(x)$ $=\infty$ for $x<0$. Such a system is described by a twodimensional Hamiltonian of the form

$$
H=H_{0}+H_{\text {SOI }}+V(x)
$$

where the Hamiltonian of free particle in a magnetic field is $H_{0}=\left(\vec{\pi}^{2} / 2 m^{*}\right) \hat{\tau}$ and the Rashba SOI Hamiltonian $H_{\text {SOI }}$ $=\alpha_{R}\left(\pi_{x} \hat{\sigma}_{y}-\pi_{y} \hat{\sigma}_{x}\right),{ }^{24} m^{*}$ denotes the electron effective mass, $\vec{\pi}=\vec{p}-(e / c) \vec{A}$ the kinetic momentum with $\vec{p}=-i \hbar \vec{\nabla}$. We choose the Landau gauge so that the components of vector potential are $\vec{A}=\left(0, x B_{0}, 0\right)$. The unity matrix $\hat{\tau}$ and the Paulispin matrices $\hat{\sigma}$ act in the spin space. It is assumed that electrons are confined to the lowest-energy subband in the $z$ direction.

Using the ansatz $\Psi(x, y)=e^{i k_{y} y} \chi_{k_{y}}(x)$, we can reduce the two-dimensional Schrödinger equation $H \Psi=E \Psi$ to the onedimensional problem where $E$ is the electron total energy and $k_{y}$ the electron momentum in $y$ direction. Then the electron wave function $\chi_{k_{y}}(x)$ in $x$ direction should satisfy the following equation 


$$
\begin{aligned}
& \left\{\left[\frac{d^{2}}{d x^{2}}+\nu+\frac{1}{2}-\frac{\left[x-X\left(k_{y}\right)\right]^{2}}{4}\right] \hat{\tau}\right. \\
& \left.\quad+\gamma\left[i \frac{d}{d x} \hat{\sigma}_{y}-\frac{x-X\left(k_{y}\right)}{2} \hat{\sigma}_{x}\right]\right\} \chi_{k_{y}}(x)=0,
\end{aligned}
$$

where the effective potential $V_{\text {eff }}(x)=\left[x-X\left(k_{y}\right)\right]^{2} / 4$ in $x$ direction depends on the wave vector $k_{y}$. In Eq. (2) we express the energy $E \rightarrow(\nu+1 / 2) \hbar \omega_{B}$ in units of the cyclotron energy, $\hbar \omega_{B} \equiv \hbar e B_{0} / m^{*} c$, and the length $x \rightarrow x l_{B} / \sqrt{2}$ in the magnetic length, $l_{B} \equiv \sqrt{\hbar c / e B_{0}}$. We introduce also the dimensionless SOI coupling constant $\gamma=\sqrt{2} \alpha_{R} / v_{B}$ with the cyclotron velocity $v_{B}=\hbar^{2} / m^{*} l_{B}$ and the dimensionless coordinate of the center of orbital rotation $X\left(k_{y}\right)=\sqrt{2} k_{y} l_{B}$. Taking into account explicitly that the eigenstates of Eq. (2) are spinors

$$
\chi_{k_{y}}(x)=\left|\begin{array}{c}
\chi_{1 k_{y}}\left[x-X\left(k_{y}\right)\right] \\
\chi_{2 k_{y}}\left[x-X\left(k_{y}\right)\right]
\end{array}\right|,
$$

we write the Schrödinger equation in the following compact form:

$$
\left(\begin{array}{ll}
h_{\nu} & h_{+} \\
h_{-} & h_{\nu}
\end{array}\right)\left|\begin{array}{l}
\chi_{1 k_{y}}(\xi) \\
\chi_{2 k_{y}}(\xi)
\end{array}\right|=0 .
$$

Here we introduce the following operators:

$$
\begin{gathered}
h_{\nu}=\left(\frac{d^{2}}{d \xi^{2}}+\nu+\frac{1}{2}-\frac{\xi^{2}}{4}\right), \\
h_{ \pm}=-\gamma\left[\frac{\xi}{2} \mp \frac{d}{d \xi}\right] .
\end{gathered}
$$

The system of equations, obtained from Eq. (4) has to be solved under the boundary conditions $\chi_{k_{y}}(x) \rightarrow 0$ when $x$ $\rightarrow 0$ and $+\infty$. In the absence of SOI, $h_{ \pm}=0$, the solution is given in terms of the parabolic cylindrical functions, $D_{\nu}(x)$. In the presence of SOI we search the bulk solution of matrix Eq. (4) as $\chi_{1 k_{y}}(\xi)=a D_{\mu}(\xi)$ and $\chi_{2 k_{y}}(\xi)=b D_{\mu-1}(\xi)$ where $a$ and $b$ are the $x$-independent spinor coefficients and $\mu$ is an arbitrary index, different from $\nu$. Making use of the following recurrent properties of the parabolic cylindrical functions:

$$
\begin{aligned}
h_{\nu} D_{\mu}(\xi) & =(\nu-\mu) D_{\mu}(\xi), \\
h_{ \pm} D_{\mu}(\xi) & =-\gamma\left\{\begin{array}{c}
D_{\mu+1}(\xi) \\
\mu D_{\mu-1}(\xi)
\end{array},\right.
\end{aligned}
$$

we obtain

$$
\begin{gathered}
\mu_{ \pm}(\nu, \gamma)=\nu+\frac{1}{2}+\frac{\gamma^{2}}{2} \pm \sqrt{\nu \gamma^{2}+\frac{1}{4}\left(1+\gamma^{2}\right)^{2}}, \\
c_{ \pm}(\nu, \gamma)=-\frac{1}{\gamma}\left[\frac{1}{2}+\frac{\gamma^{2}}{2} \pm \sqrt{\nu \gamma^{2}+\frac{1}{4}\left(1+\gamma^{2}\right)^{2}}\right]
\end{gathered}
$$

where $c_{ \pm}=b_{ \pm} / a_{ \pm}$. Thus, the two independent bulk solutions of Eq. (4) are given by the spinor wave functions

$$
\chi_{k_{y}^{ \pm}}^{ \pm}(\xi)=a_{ \pm}\left|\begin{array}{c}
D_{\mu_{ \pm}(\nu, \gamma)}(\xi) \\
c_{ \pm} D_{\mu \pm(\nu, \gamma)-1}(\xi)
\end{array}\right| .
$$

The normalization of the wave functions $\int d \xi \chi_{k_{y}}^{\dagger}(\xi) \chi_{k_{y}}(\xi)=1$ gives the amplitude of eigenstates

$$
a_{ \pm}=\left\{\int d \xi\left[\left|D_{\mu_{ \pm}}(\xi)\right|^{2}+\left|c_{ \pm}\right|^{2}\left|D_{\mu_{ \pm}-1}(\xi)\right|^{2}\right]\right\}^{-1 / 2} .
$$

It is easy to see that in the limit of vanishing SOI, $\gamma \rightarrow 0$, we have $a_{+} \rightarrow 0$ and $b_{-} \rightarrow 0$ and recover the usual edge states, which are doubly degenerated with respect to the spin

$$
\chi_{k_{y}}^{+}(x) \sim D_{\nu}(x)\left|\begin{array}{l}
1 \\
0
\end{array}\right| \text { and } \chi_{k_{y}}^{-}(x) \sim D_{\nu}(x)\left|\begin{array}{l}
0 \\
1
\end{array}\right| \text {. }
$$

On the other hand the solution (11) for sufficiently large values of $X\left(k_{y}\right)$ describes quasibulk Landau states so that the index $\mu_{ \pm}(\nu, \gamma)$ differs only exponentially from the Landau index $l=0,1,2 \ldots$ and the parabolic cylindric functions are given by their asymptotics Hermite polynomials, $D_{l}(\xi)$ $=2^{-l / 2} \exp \left(-\xi^{2} / 4\right) H_{l}(\sqrt{\xi / 2})$. In the limit of $X\left(k_{y}\right) \rightarrow \infty$ taking $\mu_{ \pm}(\nu, \gamma)=l$ in Eqs. (9) and (10), one can exactly reproduce the spectrum and the wave functions of the bulk dispersionless Landau levels, renormalized by the SOI for $l=1,2 \ldots$ (Ref. 24-28)

$$
\begin{gathered}
E_{l}^{ \pm}(\gamma)=\left(l \pm \sqrt{\frac{1}{4}+l \gamma^{2}}\right) \hbar \omega_{B}, \\
c_{ \pm}(\nu, \gamma)=-\frac{1}{\gamma}\left(\frac{1}{2} \mp \sqrt{\frac{1}{4}+l \gamma^{2}}\right) .
\end{gathered}
$$

As usual the $l=0$ Landau level remains not perturbed by the spin-orbit coupling. In order to obtain the spectrum of the spin edge states we require vanishing of the electron wave function Eq. (11) at $x=0$. The energy, $E$, obtained from the vanishing conditions for both spinor components at $x=0$, should be the same. As seen, however, the different spinor components of the bulk solution Eq. (11) are given by the parabolic cylindric functions with different indices, which makes impossible their vanishing simultaneously. To satisfy the boundary conditions, we construct a linear combination of the two independent bulk solutions as

$$
\psi_{k_{y}}(\xi)=\alpha \chi_{k_{y}}^{+}(\xi)+\beta \chi_{k_{y}}^{-}(\xi)
$$

and choose the coefficients $\alpha$ and $\beta$ so that the components of the new spinor wave function $\psi_{k_{y}}(\xi)$ vanish at $x=0$. The eigenvalue problem for $\alpha$ and $\beta$ has a solution if the respective determinant vanishes at $x=0$. This leads to the following exact dispersion equation:

$$
\begin{aligned}
& c_{-} D_{\mu_{+}}\left[-X\left(k_{y}\right)\right] D_{\mu_{-}-1}\left[-X\left(k_{y}\right)\right] \\
& \quad=c_{+} D_{\mu_{-}}\left[-X\left(k_{y}\right)\right] D_{\mu_{+}-1}\left[-X\left(k_{y}\right)\right]
\end{aligned}
$$

for the spin edge states with the wave functions 

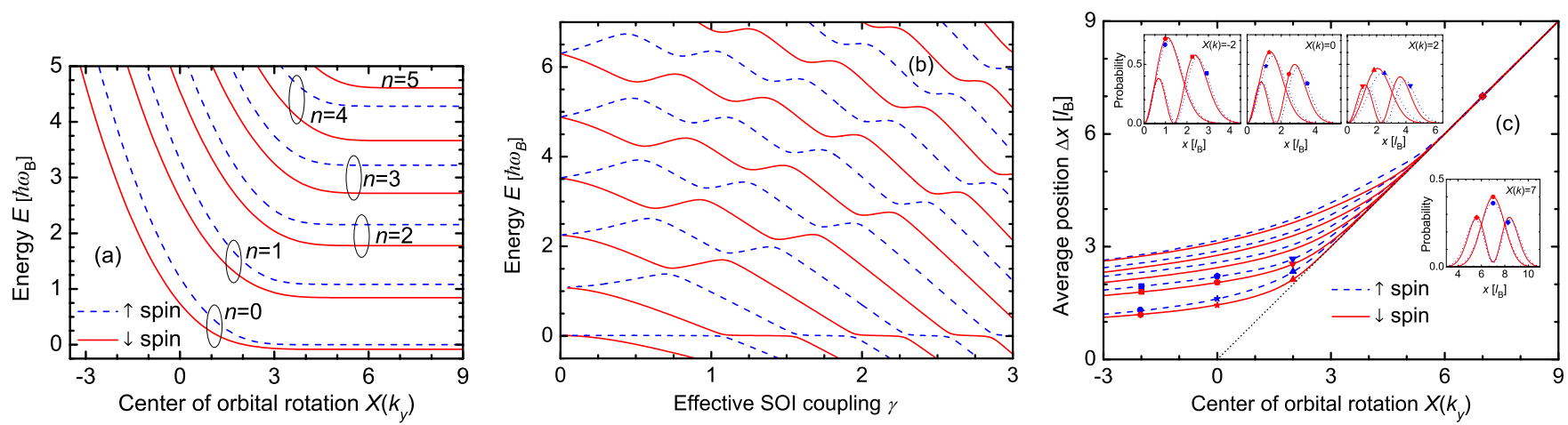

FIG. 1. (Color online) The energy spectrum of spin edge states as a function (a) of the momentum $k_{y}$ for $\gamma=0.3$ and (b) of the effective SOI coupling $\gamma$ (or that is the same as a function of $B^{-1 / 2}$ ) for $X\left(k_{y}\right)=3$. The dashed and solid curves correspond to the up and down spins. (c) The particle average position as a function of $k_{y}$ for $\gamma=0.3$. The probability density in the respective symbol positions is shown in the insets.

$$
\psi_{k_{y}}(\xi)=\alpha\left|\begin{array}{c}
D_{\mu_{+}}(\xi)-r D_{\mu_{-}}(\xi) \\
c_{+} D_{\mu_{+}-1}(\xi)-r c_{-} D_{\mu_{-}-1}(\xi)
\end{array}\right| .
$$

Here $r=D_{\mu_{+}}\left[-X\left(k_{y}\right)\right] / D_{\mu_{-}}\left[-X\left(k_{y}\right)\right]$ and $\alpha$ is obtained from the normalization of the wave functions. Recall that the dependence on energy $E=(\nu+1 / 2) \hbar \omega_{B}$ manifests itself via the functions $\mu_{ \pm}(\nu, \gamma)$ and $c_{ \pm}(\nu, \gamma)$, given by Eqs. (9) and (10). The dispersion relation Eq. (17) is quadratic with respect to the parabolic cylindric functions, therefore for a given band index, $n$, it has two solutions, $E_{s n}\left(k_{y}\right)$, where $s=\uparrow$ and $\downarrow$ corresponds to the spin edge states with the two spinor wave functions

$$
\psi_{k_{y}}^{\uparrow \downarrow}(\xi)=\left.\psi_{k_{y}}(\xi)\right|_{E=E_{\uparrow, \downarrow n}\left(k_{y}\right)} .
$$

\section{ENERGY SPECTRUM OF SPIN EDGE STATES AND SPIN CURRENT}

Further we carry out the actual calculations of the spectrum of spin edge states, the average components of spins, and the spin-current components, carried by the skipping orbits along the edges of 2DES. In the presence of a perpendicular magnetic field, the efficiency of SOI is determined by the dimensionless coupling constant $\gamma$, which is inversely proportional to $\sqrt{B_{0}}$. Therefore, the SOI effect is significant in weak magnetic fields unless temperature fluctuations smear magnetic-quantization effects. In such fields the Zeeman effect is small ${ }^{21}$ and we do not consider it here. We carry out the actual calculations for $B_{0}$ corresponding to the cyclotron splitting about $5 \mathrm{~K}$. In GaAs with the electron effective mass $m^{*}=0.067 m_{0}$ such a cyclotron splitting is achieved for $B_{0} \approx 0.25 \mathrm{~T}$ and taking the Rashba coupling $\alpha_{R} \approx 4.72 \mathrm{meV} \AA$, we have $\gamma=0.03$. For such a coupling the spin-orbit effects in the edge state spectrum should be hardly visible. The situation is favorable in InAs where the Rashba coupling is larger, $\alpha_{R} \approx 112.49 \mathrm{meV} \AA .{ }^{1}$ Despite the smaller effective mass, $m^{*}=0.026 m_{0}$, in weak fields about $B_{0}$ $=0.1 \mathrm{~T}$, we have $\gamma=0.45$. As we see below such a coupling results in essential modifications in the spectrum and transport of spin edge state, measurable in experiment.
In Fig. 1(a) we plot the energy spectrum of spin edge states, $E_{s n}\left(k_{y}\right)$, as a function of momentum $k_{y}$, which we obtain by solving the dispersion Eq. (17). It is seen that for a given quantum number $n$ there are two spin-resolved magnetic edge states, $E_{\downarrow n}\left(k_{y}\right)$ and $E_{\uparrow n}\left(k_{y}\right)$. Both branches show monotonic behavior in the whole range of $k_{y}$ variation. For large positive values of $k_{y}$, the energy of spin edge states is given by the spin-split quasibulk Landau levels, renormalized by SOI, while at negative values of $k_{y}$ the spectrum describes the current-carrying edge channels. The spin splitting of edge states increases with the main quantum number $n$. At the stronger effective SOI coupling the spectrum shows well-pronounced anticrossings. The development of the anticrossings can be traced clearly in Fig. 1(b) where we calculate the energy of spin edge states versus $\gamma$ or, what is the same, versus $1 / \sqrt{B_{0}}$. These anticrossings in the energy spectrum result in additional structures of the spin current versus the Fermi energy. In Fig. 1(c) we plot the average transverse position of the spin edge states from the boundary of 2DES as a function of their center of orbital motion, defined as

$$
\Delta x_{s n}\left(k_{y}\right)=\int_{0}^{\infty} d x x\left|\psi_{k_{y}}\left[x-X\left(k_{y}\right)\right]\right|_{E=E_{s n}\left(k_{y}\right)}^{2} .
$$

It is seen that except for large positive values of $k_{y}$, the position of skipping orbits takes spin-resolved values so that the up- and down-spin-edge states are separated in space. Notice this effect of the SOI-induced spatial separation is missed in the approximation, adopted in Ref. 17. The differences in the probability density for different spins and wave vectors of the first two bands are clearly shown in the insets. As seen from the lower inset, the probability density for different bands and spins differs even at large positive values of $k_{y}$. In this limit, however, irrespective the quantum number $n$ and the spin orientation, the particle average thickness is the same and varies linearly with its center of orbital motion. This is because in the quasibulk Landau states far from the interface, electrons oscillate symmetrically with respect to the guiding center, $X\left(k_{y}\right)$, independent of the spin and band index.

From the obtained spectrum we calculate the corresponding group velocities along $y$ direction, $v_{s n}\left(k_{y}\right)$ 


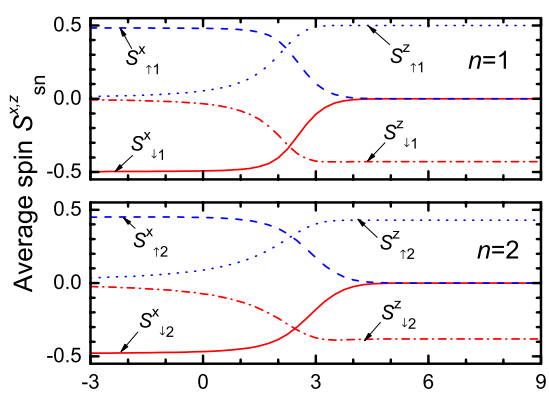

(a)

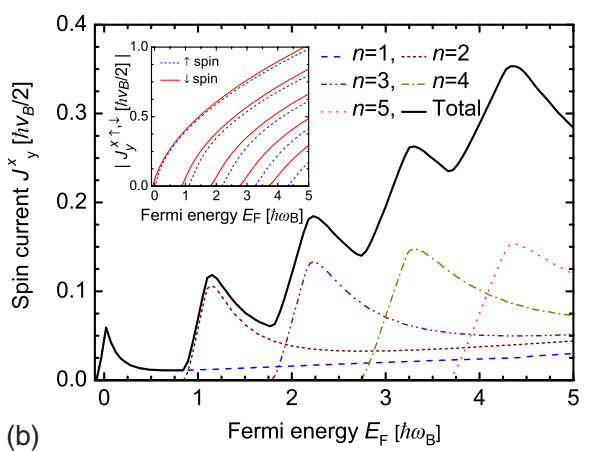

(b)

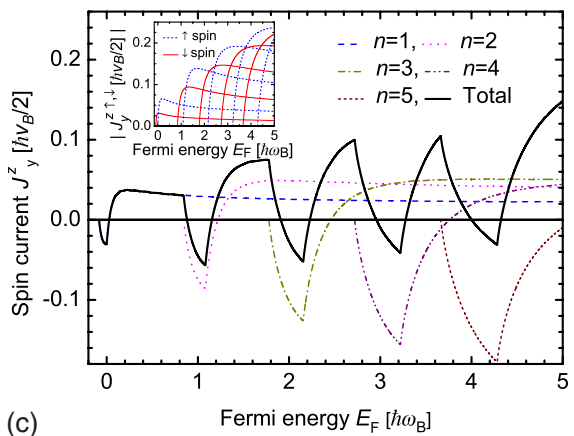

FIG. 2. (Color online) (a) The $x$ and $z$ components of average spins in units of $\hbar$ as a function of $X\left(k_{y}\right)$ for the first two bands $n=1,2$ and for $\gamma=0.3$. (b) The $x$ component and (c) the $z$ component of the net-spin current (solid curve) versus the Fermi energy. The dashed and dotted curves plot the separate contributions to the spin current made by the spin-up and spin-down states together for each band $n$. Insets show the absolute value of the separate up- and down-spin-current contributions.

$=\partial E_{s n}\left(k_{y}\right) / \partial \hbar k_{y}\left(v_{x} \equiv 0\right.$ along $x$ direction $)$ as well as the average spin components along $x, z$ directions

$$
S_{s n}^{x, z}\left(k_{y}\right)=\left.\frac{\hbar}{2} \int_{0}^{\infty} d x \psi_{k_{y}}^{\dagger}(x) \hat{\sigma}_{x, z} \psi_{k_{y}}(x)\right|_{E=E_{s n}\left(k_{y}\right)} .
$$

Because the transverse wave functions are real, the $y$ component of the spin vanishes identically, $S_{s n}^{y}\left(k_{y}\right) \equiv 0$. In Fig. 2(a) we plot $S_{s n}^{x, z}\left(k_{y}\right)$ as a function of $X\left(k_{y}\right)$ for the first two bands. At large positive values of $k_{y}$ when electrons are far from the hard wall, the spins are mainly aligned along $z$ axes. This is because in the quasibulk Landau states electrons have no preferential direction in the $(x, y)$ plane of their cyclotron rotation. In the opposite limit of negative $k_{y}$, the edge channels are formed and the spins are mainly aligned in $x$ direction, perpendicular to the $y$ direction of electron propagation. Notice that due to the spin splitting the absolute values of the average spin components do not equal in the up and down states and this asymmetry becomes stronger with the band index $n$.

In Figs. 2(b) and 2(c) we calculate the $x$ and $z$ components of the net-spin current along $y$ direction, defined as

$$
J_{y}^{x, z}\left(E_{F}\right)=\left.\sum_{s, n} S_{s n}^{x, z}\left(k_{y}\right) v_{s n}\left(k_{y}\right)\right|_{E_{s n}\left(k_{y}\right)=E_{F}} .
$$

We use this definition of the spin current, which is widely accepted and intuitive from the physical point of view. Notice that according to some recent suggestions, ${ }^{29,30}$ the standard definition of spin current (which is the one we use here) is a proper definition and there is no need for other definitions. ${ }^{31-33}$ It is seen that $J_{y}^{x}$ exhibits regular peaks as a function of the Fermi energy. Such an oscillatory behavior is due to the SOI-induced splitting in the edge state spectrum. For a given quantum number $n$ the splitting always creates a narrow energy region near the peak position [see the inset in Fig. 1(b)] where only the $\downarrow$ spin states contribute positively to the spin current. With an increase in the Fermi energy the $\uparrow$ spin edge state starts to contribute negatively at the position of $\uparrow$ spin Landau level. In this region the exponential increase in the velocity ${ }^{34}$ of the $\uparrow$ spin edge state with the energy results in a sharp peak of the spin current. With a further increase in the Fermi energy the $\downarrow$ and $\uparrow$ spin edge states of the next $n+1$ band start to contribute similarly but with stronger amplitudes because of the spin-splitting enhancement with $n$. Thus the peaks of the spin current are imposed against the monotonic background and have a period, determined by the cyclotron energy. The latter is renormalized in the presence of SOI, as seen in Fig. 1(a) in the limit of $k_{y} \rightarrow \infty$.

As seen in Fig. 2(c) the $z$-spin current $J_{y}^{z}$ changes its sign, in addition to its peaked behavior: due to an interplay between the average spin $S_{s n}^{z}\left(k_{y}\right)$ and the velocity $v_{s n}\left(k_{y}\right)$, the spin current is negative near the Landau levels and positive between them. In this case, at high energies corresponding to large negative values of $k_{y}$ in each band $n$ [cf. Fig. 1(a)], the large values of $v_{s n}\left(k_{y}\right)$ are compensated by the small values of $S_{s n}^{z}\left(k_{y}\right)$ [cf. Fig. 2(a)]. Therefore the overall monotonic increase in $J_{y}^{z}$ with $E_{F}$ becomes less pronounced.

\section{SUMMARY}

In conclusion, we present an exact analytical solution to the spin edge states, induced by the combined effect of the Rashba SOI and of the hard-wall confining potential. The exact solution of the problem allows its deeper intuitive understanding and can be a strong input in studying the spin transport through edge channels. We find that due to SOI the spin edge states are resolved not only in the energy but are also separated in space. In the bulk of sample the electron spin is mainly aligned along the magnetic field while near the hard wall the spin becomes mainly perpendicular to the edge state propagation direction. The magnitude of spin components is asymmetric in the up and down states. We show that the spin-current components exhibit oscillations versus the Fermi energy. The predicted oscillations, with a period determined by the renormalized cyclotron energy, can serve as an effective tool to control the spin motion in spintronic devices.

\section{ACKNOWLEDGMENTS}

We thank J. Fabian and G. Vignale for useful discussions and acknowledge support from the Volkswagen Foundation, EU under Grant No. PIIF-GA-2009-235394, SFB under Grant No. 689, and ANSEF under Grant No. PS-1576. 
*samvel.badalyan@physik.uni-regensburg.de

${ }^{1}$ J. Fabian, A. Matos-Abiague, C. Ertler, P. Stano, and I. Žutić, Acta Phys. Slov. 57, 565 (2007).

${ }^{2}$ I. Žutić, J. Fabian, and S. Das Sarma, Rev. Mod. Phys. 76, 323 (2004).

${ }^{3}$ S. A. Wolf, D. D. Awschalom, R. A. Buhrman, J. M. Daughton, S. von Molnár, M. L. Roukes, A. Y. Chtchelkanova, and D. M. Treger, Science 294, 1488 (2001).

${ }^{4}$ M. I. D’yakonov and V. Yu. Kachorovskii, Sov. Phys. Semicond. 20, 110 (1986).

${ }^{5}$ Y. K. Kato, R. C. Myers, A. C. Gossard, and D. D. Awschalom, Science 306, 1910 (2004).

${ }^{6}$ C. P. Weber, N. Gedik, J. E. Moore, J. Orenstein, J. Stephens, and D. D. Awschalom, Nature (London) 437, 1330 (2005).

${ }^{7}$ S. M. Badalyan, C. S. Kim, and G. Vignale, Phys. Rev. Lett. 100, 016603 (2008).

${ }^{8}$ B. A. Bernevig, J. Orenstein, and S. C. Zhang, Phys. Rev. Lett. 97, 236601 (2006).

${ }^{9}$ S. M. Badalyan, A. Matos-Abiague, G. Vignale, and J. Fabian, Phys. Rev. B 79, 205305 (2009).

${ }^{10}$ R. B. Laughlin, Phys. Rev. B 23, 5632 (1981).

${ }^{11}$ B. I. Halperin, Phys. Rev. B 25, 2185 (1982).

${ }^{12}$ A. H. MacDonald and P. Streda, Phys. Rev. B 29, 1616 (1984).

${ }^{13}$ S. M. Badalian, Y. B. Levinson, and D. L. Maslov, JETP Lett. 53, 619 (1991).

${ }^{14}$ G. Müller, D. Weiss, A. V. Khaetskii, K. von Klitzing, S. Koch, H. Nickel, W. Schlapp, and R. Lösch, Phys. Rev. B 45, 3932 (1992).

${ }^{15}$ A. Khaetskii and K. A. Matveev, J. Phys.: Condens. Matter 4, 3491 (1992).

${ }^{16}$ Y. Ji, Y. Chung, D. Sprinzak, M. Heiblum, D. Mahalu, and H. Shtrikman, Nature (London) 422, 415 (2003).

${ }^{17}$ M. G. Pala, M. Governale, U. Zulicke, and G. Iannaccone, Phys.
Rev. B 71, 115306 (2005).

${ }^{18}$ Y.-J. Bao, H.-B. Zhuang, S.-Q. Shen, and F.-C. Zhang, Phys. Rev. B 72, 245323 (2005).

${ }^{19}$ J. Wang, H. B. Sun, and D. Y. Xing, Phys. Rev. B 69, 085304 (2004).

${ }^{20}$ A. Reynoso, G. Usaj, M. J. Sanchez, and C. A. Balseiro, Phys. Rev. B 70, 235344 (2004).

${ }^{21}$ S. Debald and B. Kramer, Phys. Rev. B 71, 115322 (2005).

${ }^{22}$ A. T. Ngo, J. M. Villas-Bôas, and S. E. Ulloa, Phys. Rev. B 78, 245310 (2008).

${ }^{23}$ H. Su and B.-Y. Gu, Phys. Lett. A 341, 198 (2005).

${ }^{24}$ E. I. Rashba, Sov. Phys. Solid State 2, 1109 (1960); Y. A. Bychkov and E. I. Rashba, Pis'ma Zh. Eksp. Teor. Fiz. 39, 66 (1984).

${ }^{25}$ J. Schliemann, J. C. Egues, and D. Loss, Phys. Rev. B 67, 085302 (2003).

${ }^{26}$ S. Q. Shen, M. Ma, X. C. Xie, and F. C. Zhang, Phys. Rev. Lett. 92, 256603 (2004).

${ }^{27}$ S. Q. Shen, Y.-J. Bao, M. Ma, X. C. Xie, and F. C. Zhang, Phys. Rev. B 71, 155316 (2005).

${ }^{28}$ E. Lipparini, M. Barranco, F. Malet, M. Pi, and L. Serra, Phys. Rev. B 74, 115303 (2006).

${ }^{29}$ I. V. Tokatly, Phys. Rev. Lett. 101, 106601 (2008).

${ }^{30}$ Q.-F. Sun, X. C. Xie, and J. Wang, Phys. Rev. B 77, 035327 (2008).

${ }^{31}$ A. Vernes, B. L. Györffy, and P. Weinberger, Phys. Rev. B 76, 012408 (2007).

${ }^{32}$ R. Shen, Y. Chen, Z. D. Wang, and D. Y. Xing, Phys. Rev. B 74, 125313 (2006).

${ }^{33}$ J. Shi, P. Zhang, D. Xiao, and Q. Niu, Phys. Rev. Lett. 96, 076604 (2006).

${ }^{34}$ S. M. Badalyan and F. M. Peeters, Phys. Rev. B 64, 155303 (2001). 